PART 4

STELLAR ACTIVITY OF THE SOLAR TYPE 


\title{
STELLAR ACTIVITY OF THE SOLAR TYPE \\ OBSERVATIONAL ASPECTS
}

\author{
G. GODOLI
}

Catania Astrophysical Observatory and Astronomical Institute, University of Catania, Italy

\section{Introduction}

By the expression 'stellar activity of the solar type' we mean the manifestations of stellar activity determined by a mechanism of the same kind as that producing the solar activity.

As we already know, this mechanism should consist in the interplay of solar poloidal magnetic fields, differential rotation and convection. Gravitational actions by planets and interactions with diffuse matter could also play as triggering agents. It is evident that if this mechanism acts on different stars in different physical conditions it could give rise to quantitatively and qualitatively different phenomena. Vice versa, from the analysis and interpretation of these phenomena, knowing the physical conditions in which they take place, we can hope to deduce new information on the mechanism involved.

Gershberg (1975) notes that since we have no complete physical theory either for the totality of events that take place in a solar active region, or for the mechanism involved in solar activity as a whole, somebody could argue that analogies between solar and stellar events do not contain new information. Wittily Gershberg points out that also Newton found only (!) some analogies between the falling of an apple and the motion of the Moon!

The problem of stellar activity of the solar type was thoroughly investigated by Unsöld in the second edition of his Physik der Sternatmosphären (1955) and was successively taken up again by Smith and Smith in their book on solar flares (1963) and by Bray and Loughhead in their book on sunspots (1964). In 1967 a wide research programme on stellar activity of the solar type was undertaken at the Catania Astrophysical Observatory (Godoli, 1967). In 1968 a review of the problem and preliminary results collected at Catania were presented by the writer at the Nobel Symposium. Problems connected with stellar activity of the solar type were reviewed by Oster in the volume recently dedicated to Albrecht Unsöld (1975). Finally, a thorough analysis on the analogies between stellar and solar flares and between stellar and solar flare activities was presented by Gershberg (1975) at the IAU Symposium No. 67 on 'Variables in Relation to the Evolution of Stars and Stellar Systems'.

We can assert that in the last few years the problem of stellar activity of the solar type has been an aspect of the increasing convergence in the fields of stellar and solar physics.

In this review we shall summarize the latest results in this field.

For the sake of completeness we shall also briefly recall information reported in the quoted literature. 


\section{The Sun as a Variable Star}

\subsection{INTRODUCTION}

Before trying to describe stellar observational facts that could be determined by a mechanism of the same kind as that producing the solar activity, let us briefly consider how the manifestation of solar activity would appear at stellar distances.

\subsection{SOLAR ROTATION AND MAGNETIC FIELD}

As Howard (1972) pointed out, if the Sun were observed as a distant star its rotation rate could be detected only at the very highest stellar spectroscopic dispersion and the $5 \%$ variation observed in the Sun rotation rate would pass entirely undetected.

In 1967 Bumba et al., using, instead of the solar image, a parallel beam from the coelostat mounting falling on the magnetograph slit, showed that the magnetic field of the Sun observed as a star is about two orders of magnitude weaker than the smallest fields that could be detected in stellar observations at that time.

In 1967 Severny started more or less systematic observations of the magnetic field of the Sun observed as a star. The results of these observations and their relationships to the sector structure of the interplanetary magnetic field are well known to all solar physicists. We only recall the explanation given by Tuominen (1970) of the fact that the magnetic flux from sunspots is in antiphase with the fluctuations of the measured total field (Severny, 1969). According to Tuominen, since the following part of a bipolar magnetic region disintegrates much faster than the leading one, the following part has a greater influence on the observed large scale fields. On the contrary, when determining the magnetic flux from sunspots, the leader spots are more effective. Tuominen also suggests that the long lifetime of the large scale magnetic region can be explained on the basis of the occurrence of the activity complexes (Bumba and Howard, 1965).

\subsection{SOlAR LUMINOSITY}

The problem of the variations of the so-called solar constant is very old and intricate. We recall here only the attempts made for collecting information on the variation of the solar constant from the amount of light reflected by the planets Uranus and Neptune (Johnson and Iriarte, 1959; Serkowski, 1961; Jerzykiewicz and Serkowski, 1966; Albrecht et al., 1969).

\subsection{SUNSPOTS}

The largest sunspot group ever recorded, observed in 1947, had a maximum area of a little more than a hundredth of the solar disk. If we consider this group completely dark, it would give rise, owing to the solar rotation, to a light variation of about one hundredth of magnitude. This value falls within the possibility of photoelectric photometry.

\subsection{Photospheric AND Chromospheric faCUlaE}

A variation of the same order as above would be given by photospheric faculae. 
Since the presence of magnetic fields in the solar atmosphere is related to the $\mathrm{H}_{2}$ and $\mathrm{K}_{2}$ emission component of the $\mathrm{H}$ and $\mathrm{K}$ lines of $\mathrm{Ca} I \mathrm{It}$ is obvious to expect variation of these emissions if we observe the Sun as a star.

Actually Sheeley (1967) and Bumba and Růžičková-Topolová (1967) found, independently, with different methods, variations of the intensity of $\mathrm{K}_{2}$ emission averaged on the disc of as much as $15 \%$ during a solar rotation and of $40 \%$ during a solar cycle, whereas Bappu (1967) found these values too optimistic.

\subsection{SolAR FLARES}

Optical white light flares would give rise to variations at the limit of the possibilities of photoelectric photometry. However, as early as 1950 , Greenstein pointed out that bright solar flares could double the brightness of an $\mathrm{M}$ dwarf: moreover a flare on such a star would be more prominent in the violet because of the steep drop in the stellar continuum.

Some years later, in 1959, Schatzman showed that the flux of solar type II bursts could be detected with the most sensitive radiotelescope of that time even at stellar distances.

\section{Stellar Flares}

\subsection{INTRODUCTION}

The task of picking out, from the multiplicity of observational stellar facts, those that could be determined by a mechanism of the same kind as that producing the solar activity is extremely intricate.

We shall first analyse stellar flares since these phenomena have many common features with solar flares. Speaking of stellar flares we will be in the wake of the problems concerning photospheric, chromospheric and coronal stellar activity.

Many reviews are available on the problem of stellar flares. We recall, among the most recent ones those of Gershberg (1971, 1975), Lovell (1971), Ambartsumian and Mirzoyan (1971), Gershberg and Pikelner (1972), Kunkel (1975), Gershberg and Lund (1975). Therefore we shall only summarize the most relevant information from our point of view (for more details and references we refer to the quoted reviews) and present the latest results. We shall confine ourselves to only UV Ceti-type stars although we know that flare activity can be present in stars of other types too.

UV Ceti-type stars are red dwarfs with spectra showing emission lines (dKe and $\mathrm{dMe}$ ). Surface temperatures are less than $3500 \mathrm{~K}$; luminosities are of the order of $10^{-5}$ solar luminosity; masses are of the order of $10^{-1}$ solar masses.

These stars undergo the most rapid variations of all eruptive variables. In a time interval ranging from a fraction of a second to several minutes, their integrated brightness sometimes increases by more than five magnitudes. The decay time is of the order of 1-60 min. Very intense and.complex events have been observed to last even a few hours. Optical observations from the $U$ - to the $V$-band show that flare amplitude decreases in going from shorter to longer wavelengths.

Typical frequency is $10^{-1}$ events per hour. 
Traditional methods of photographic and photoelectric spectroscopy cannot be used to get information on spectra of stellar flares since exposure time must be longer than the duration of flares. Image tubes must be used as light detectors.

During a flare, spectra undergo radical changes. The overall characteristics of flare spectra almost completely coincide with the characteristics of the spectra of $T$ Tauri type stars. Bright intense lines and strong continuous emission are present especially in the ultraviolet. At the beginning of the flare, hydrogen and neutral helium lines increase strongly. Simultaneously strong emission in the Balmer continuum appears. In some strong flares also the $4686 \AA$ line of the ionized helium and the $5184 \AA$ line of neutral magnesium appear. Ca II emissions increase somewhat later and to a lesser extent than the emission of the Balmer lines. After flare maximum the decay of the continuum emission is much more rapid than the decay of the line emission and the helium lines fade quicker than the hydrogen lines. Intensified calcium emission has been observed even several hours after strong flares.

We notice that spectral features, as TiO bands and the Ca I $4227 \AA$ line, characteristic of the cold stellar atmosphere, remain during flares.

We have already pointed out that as early as 1959 Schatzman showed the possibility of observing radio bursts from flare stars.

This possibility directed the attention of Lovell to these objects. Investigation began at the Mark 1250-ft radiotelescope at Jodrell Bank in 1958, September 28 and was continued at intervals until 1960, April $14.474 \mathrm{~h}$ of observations were made in the meter waveband at $240 \mathrm{MHz}$ with a technique suitable for rejecting spurious bursts of noise arising from terrestrial sources. When all doubtful cases had been excluded, 13 events with durations of several minutes were noticed. For UV Ceti, rates of occurrence of radio and optical events were closed. Notwithstanding these preliminary results simultaneous optical and radio observations were needed in order to ascertain the nature of the recorded radio events.

Many national and international campaigns have been organized since then also under the auspices of Commission 27 of the International Astronomical Union.

Up-to-date radio emission during stellar flares has been recorded in the range $20 \mathrm{~cm}-15 \mathrm{~m}$.

With a radio apparatus sensitivity of $0.3 \mathrm{f}$.u. radio flare occurrence is close to optical flare occurrence.

Observable X-ray emission during stellar flares was predicted by Gurzadian (1965, 1966b), Grindlay (1970), Gurzadian (1971) and Crammel et al. (1974).

An estimation of the upper limit of the X-ray flux from flares of UV Ceti type stars was carried out by Gershberg et al. (1969) studying the correlation between stellar flares and sudden cosmic noise absorption. No correlation was found. Supposing that the effect is smaller than the fluctuations of cosmic noise an upper limit of $10^{-4} \mathrm{erg} \mathrm{cm}^{-2} \mathrm{~s}^{-1}$ was determined.

Arrangements had been made to observe YZ CMi in January and February 1971 simultaneously in the optical, radio and X-range with the satellite SAS-A launched in 1970, December 12. Unfortunately flare star observation from SAS-A had to be cancelled due to some instrumental problems.

At the 14th International Cosmic Ray Conference, Grindlay and Heise (1975) presented a paper describing observations that allowed the detection of X-ray emission from flare stars for the first time (Heise et al., 1975). 
Observations were made by soft and hard X-ray detectors of the Space Research Laboratory at Utrecht and the Center for Astrophysics at Cambridge Mass. which were on board the Astronomical Netherlands Satellite (ANS). Utrecht detectors are sensitive in the ranges $0.2-0.28 \mathrm{keV}$ and $1-7 \mathrm{keV}$; Cambridge detectors in the range $1-30 \mathrm{keV}$.

The stars YZ CMi and UV Cet were observed for the periods 1974 October 19-22 and 1975 January 3-9 respectively.

A total of 300 min of good quality data with low background was obtained on YZ CMi with the satellite continuously pointed to the source for typically 5-12 min per orbit. Optical and radio monitoring was conducted for only about a third of the period of X-observation. No optical or radio flares were detected.

On October 19 at 2005 UT an X-ray flare was observed with the soft and medium energy detectors. No increase in count rate was observed in the Cambridge detector, probably for pointing reasons that cannot be verified. Unfortunately no optical or radio coverage was available.

The total duration of the flare was $6 \mathrm{~min}$ at $0.2-0.28 \mathrm{keV}$ and $1.5 \mathrm{~min}$ at $1-7 \mathrm{keV}$. The total energy released in the range $0.2-0.28 \mathrm{keV}$ was $4.2 \pm 0.3 \times 10^{31} \mathrm{erg}$ with a peak luminosity of $2.5 \pm 0.4 \times 10^{29} \mathrm{erg} \mathrm{s}^{-1}$ and in the range $1-7 \mathrm{keV}$ was $1.9 \pm 0.4 \times$ $10^{32}$ with a peak luminosity of $3.6 \pm 0.7 \times 10^{30} \mathrm{erg} \mathrm{s}^{-1}$.

A total of 316 min of good quality data with low background was obtained on UV Cet. During this time four events were observed in the optical range.

On January 8, at 011712 UT a large optical flare was observed by Kunkel and Zarate (1975): its peak luminosity was $>1.5 \times 10^{30} \mathrm{erg} \mathrm{s}^{-1}$. X-ray measurements started $28^{\mathrm{s}}$ later at 011740 with a strong enhancement in the $0.2-0.28 \mathrm{keV}$. X-ray flares lasted $48 \mathrm{~s}$. The minimal total energy released in this range was $2.9 \pm 0.6 \times$ $10^{30} \mathrm{erg}$ with an average luminosity of at least $6.1 \pm 1.3 \times 10^{28} \mathrm{erg} \mathrm{s}^{-1}$. During this time the upper limit in the Utrecht medium energy detector and in the Cambridge detector was respectively $1.8 \times 10^{30}$ and $1.3 \times 10^{30} \mathrm{erg} \mathrm{s}^{-1}$.

During the other three optical events $\mathrm{X}$ monitoring was conducted with negative results. Only the upper limit of the $4 \sigma$ level for the X-ray flux was given.

New observations of UV Ceti had been planned for the period 1975, July 4-9 (Grindlay, 1975). Unfortunately this period is not suitable for ground-based observations.

Ground-based optical and radio observations were organized by Moffett (1975a, 1975b) for autumn 1975-early winter 1976 in coordination with X-ray observations from MIT/SAS-C satellite. Observations of the flare stars UV Cet, YZ $\mathrm{CMi}$, AD Leo were planned.

Nowadays we know of hundreds of UV Ceti-type stars (including BY Dra and UVn stars) of which 39 (single stars or multiple systems) are within a distance of 25 pc from the Sun (Rodonò 1975a). As far as I know, the latest discovery of a flare star was made at Catania (Cristaldi and Rodonò, 1976) last July in the large proper motion pair G. 208-44/45 (Giglas et al., 1967) recently recognised by Harrington et al. (1974) as a nearby binary system located at $4.7 \mathrm{pc}$.

Photoelectric patrol for more than $10 \mathrm{~h}$ has been carried out, generally in the B band, for 34 stars (Rodonò, 1975a) mainly at the observatories of Armagh, Boyden, Catania, Cerro Tololo, Crimea, McDonald, Tokyo. Systematic sequential and simultaneous $U B V$, radio, and polarimetric observations have also been executed. 
Among the 39 objects within a distance of 25 pc from the Sun, $60 \%$ are binaries or multiple systems (Rodonò, 1975b). Although Rodonò (1975b) found no evidence of mutual flare triggering between components of binary systems, it is stimulating to note that a percentage of $60 \%$ could be higher than the average one (Batten, 1973) and that many of these systems have very eccentric orbits. Not so long ago there had been a belief that only the fainter components of these systems were flare stars. Nowadays flare activity on the more luminous component of visual binaries and on both of the components has been discovered in several instances.

Since $\mathrm{dK}$ and $\mathrm{dM}$ stars are intrinsically faint, UV Ceti type stars can be detected only in the neighbourhood of the Sun. Because dM stars constitute $80 \%$ of the galactic stellar population and dMe stars $5 \%$ of the dM type stars, we can argue, following Gershberg, that the flares of UV Ceti-type stars, are the most widespread kind of stellar variability.

Flare stars have not been discovered only in the neighbourhood of the Sun but also in hundreds in stellar aggregates (associations and clusters). Photographic patrol vigorously pursued particularly by Ambartsumian, Haro, Rosino and collaborators is generally used for discovery and study of flares in aggregates.

The multiple exposure technique has been employed with a time resolution of 5-10 min, inadequate to detect faint short-lived events. Recently also photoelectric methods were successfully employed by Rodonò (1974).

According to Ambartsumian and Mirzoyan (1971) T Tauri-type stars, flare stars in stellar aggregates and UV Ceti-type stars form a wide class of comparatively young, related, non-stable objects.

According to Gershberg (1975) in the light of up-to-date observations we would seemingly reject the hypothesis that UV Ceti-type stars are the later stage of $T$ Tauri-type stars. It would be better to think that the variables of both types have originated in the same volume but have different behaviours due to mass differences. For flare activation, the existence of a deep convection is important, not the age itself.

\subsection{Properties of Stellar flares}

\subsubsection{Extension}

We have various proofs that stellar flares are events localized in small regions of the stellar atmospheres.

(1) As we shall see, spectroscopic and photometric methods show that the temperature of the perturbed region must be of the order of several $10^{4} \mathrm{~K}$. If this temperature increase involves the whole stellar atmosphere, we should expect an increase in the visual surface brightness greater by one-two orders of magnitude than that observed.

(2) We have already noticed that some spectral features, which are characteristic of the cold stellar atmospheres, remain unchanged during flares. We must deduce that either the flares are localized in a small part of the stellar surface or they develop in high atmospheric layers without influencing the photosphere.

(3) Flare stars have sizes of 1-2 light seconds, whereas several flare structures have been observed to approach maxima in less than a second. 


\subsubsection{Physical Properties}

The rates of energy production during a great stellar flare are of the order of approximately $10^{25} \mathrm{erg} \mathrm{s}^{-1}$, over a bandwidth of $400 \mathrm{MHz}$, in the radio spectrum and of the order of $10^{30} \mathrm{erg} \mathrm{s}^{-1}$, over a bandwidth of $6.7 \times 10^{8} \mathrm{MHz}$ in the optical spectrum.

Spectroscopic and photometric methods show that the temperature of the perturbed region must be of the order of several $10^{4} \mathrm{~K}$ and the electron density of the order of $10^{12}-10^{14} \mathrm{~cm}^{-3}$.

Assuming localized emission, brightness temperature in radio flares at metric wavelength is of the order of $10^{16}-10^{18} \mathrm{~K}$.

Spectrographic evidences of mass motions during a flare exist in literature although they are very scanty. The most convincing observation is that of Wolf 359 (the fourth nearest star or the third nearest system) by Greenstein and Arp (1969). In an image tube spectrogram, taken in an unknown phase of a flare, the $\mathrm{K}$ line was shifted by $-59 \mathrm{~km} \mathrm{~s}^{-1}$ and the mean of about 9 hydrogen lines was changed by $-23 \mathrm{~km} \mathrm{~s}^{-1}$. Arp and Greenstein ascribed the negative shift to a hot cloud, ejected towards the observer, at velocity like that in solar spicules and less than that in eruptive prominences.

\subsubsection{Statistical Properties}

Generally the duration of a flare increases with the increase of the amplitude.

In many radio optical correlations there has been evidence that the maximum phase of the radio flare follows that of the optical flare by several minutes.

In 1972, October 11 simultaneous radio and optical recordings of a large flare on UV Ceti were obtained by Lovell et al. (1974). In this event we have a delay of about $500 \mathrm{~s}$ between the onset of the flare at optical and radio frequencies and moreover the maximum phase of the radio flare follows that of the optical flare by $11 \pm 1$ min.

Statistical investigations show that, although flares of UV Ceti-type stars occur in time, generally following the Poisson law, the number of the occurrences of very close flares is higher than expected according to the Poisson law. Also for stellar flares we could therefore have sympathetic phenomena. New information by Rodonò (1976) on this matter will be reported in this section. Also homologous phenomena could be present.

In a given star the flare frequency increases as the amplitude decreases.

The existence of a periodical or quasi-periodical time variation in the frequency of flares in-a given star is much discussed.

\subsubsection{Relationship with the Parent Star}

There appears to be in each star an upper limit to the energy radiated by a single flare and to the time averaged energy radiated by all the flares. According to Cristaldi and Rodonò (1975) the first limit is, in the $\mathrm{B}$ band, 60 times the energy emitted by the quiet star in one minute; the second one is, also according to Kunkel $(1970,1973)$, one percent of the energy radiated by the quiescent atmosphere. 
Flare frequency decreases as the stellar luminosity increases but more powerful flares are observed in more luminous stars, although there are indications that flare luminosity increases slower than star luminosity (Cristaldi and Rodonò, 1975). Due to these indications, it seems difficult to deduce information on the relationship between flare activity and luminosity of the parent star from the relationship between flare frequency and stellar luminosity.

\subsection{Physical nature of stellar flares}

A complete model of stellar flares should describe:

(1) the mechanism of storage and dissipation of the flare energy;

(2) typical behaviour of the flare light curves, colours and spectra; and

(3) regularities observed by flare statistics.

As far as storage and dissipation of the flare energy is concerned, we have three main models: the nebular model, the fast electrons model, and the shock wave model.

The nebular model elaborated by Gershberg (1967) and also by Kunkel (1967) assumes that an optical flare is due to the radiation of a hot ionized and rapidly emitting gas mass ejected by the star.

Ambartsumian showed as early as 1954 that the continuum emission, usually present in spectra of $T$ Tauri-type stars and appearing in the spectra of flare stars during the flares, should not be of thermal nature. Afterwards further evidence was obtained in favour of the non-thermal and non-synchrotron nature of the flare continuous emission.

As early as 1965 Gurzadyan assumed that 'fast electrons' i.e. non thermal, but not extremely relativistic electrons, whose energy is about $1-2 \mathrm{MeV}$, can be formed in the stars' atmospheres directly above the photosphere. The scattering of photons at such electrons is accompanied by an increase in the frequency of the photon scattered after collision (inverse Compton effect). The flare itself should be (Gurzadian, 1966a) a sudden appearance of fast electrons over the photosphere of the star. These electrons should cause a transition of the infrared quanta of the photosphere into visual and ultraviolet quanta. Naturally, assuming identical energy parameters for the electrons, the amplitude of the flare increases, in agreement with the observations, when the stellar temperature decreases. In this model polarization of the flare light is to be expected and radio emission during the flare is possible. Finally, Gurzadyan (1972) has recently pointed out that non-thermal bremsstrahlung produced by the fast electrons is practically of no significance, except for the very brightest flares observed.

According to the shock wave model of Klimishin (1970), Korovyakovskaya (1972) the flare could be due to a de-excitation of ionized hydrogen gas behind a shock front propagating in the stellar atmosphere. The Greenstein and Arp observation already discussed would suggest that a shock wave is responsible for the heating of the matter that emits radiation during the flare. Klimishin, assuming that the matter density is constant in the star atmosphere, obtained flare rise and decay times that are not in agreement with the observations. On the other hand, Korovyakovskaya was able to explain many photometric and spectroscopic characteristics of the flares assuming a 
stellar atmosphere with a density gradient and obtaining differences in the relaxation times and acceleration of the shock front when it moves into a region of lower density.

Unfortunately, till now, the available observations (mainly flare colours) do not allow a selection of a model (Cristaldi and Rodonò, 1975).

\subsection{COMPARISON OF UV CETI-FLARES WITH SOLAR FLARES}

\subsubsection{The Atmosphere of the Sun and of the UV Ceti-Stars}

Although we shall discuss afterwards the problems of the activity of stellar photospheres, chromospheres and coronae we must now point out some facts.

(1) In flare stars, as in the Sun, photospheric dark short-lived spots exist.

(2) In flare stars, as in the Sun, spot appearances show periodicities of several years.

(3) During stellar flares, as during solar flares, mass motion can develop.

(4) The flare star spectra at the minimum, i.e. outside the flares, differ from the spectra of normal dwarf stars by the presence of emission lines: as for the Sun, active stellar chromospheres are correlated with the capacity of producing flares.

(5) According to Kahn (1974) the delay between the onset of the flare at optical and radio frequencies shows that the disturbance coming from the optical flare must have spread to a corona of the star. The energy required to heat the corona would come from the mechanical energy of the convective motions in and near the photospheric layers.

\subsubsection{Analogies between Flares of UV Ceti-Type Stars and Solar Flares}

Flares of UV Ceti-type stars and solar flares have many important common features.

(1) Both phenomena are transient events.

(2) Both phenomena refer only to a small region of the stellar surface.

(3) For stellar and solar flares the ratios between rise and decay times are of the same order.

(4) Variations of spectra during stellar and solar flares are analogous.

(5) Optical thickness in $\mathrm{H} \alpha$-lines for stellar and solar flares are similar.

(6) Electron density of stellar flares is of the same order as electron density of solar flares spreading to some higher densities.

(7) Spectral behaviours of radio stellar flares and temporal relationships between optical and radio stellar flares are similar to the ones of the solar radio noise storm and sometimes of the solar type II burst. Typical velocities are $1000 \mathrm{~km} \mathrm{~s}^{-1}$.

(8) The ratio between the rates of energy production in the optical spectrum and in the radio spectrum is $10^{5}$ for stellar and solar flares.

(9) Homologous and sympathetic stellar flares can exist; we must deduce that also in flare stars persistent morphological features of the active region (most probably its magnetic field) control the flare process and that triggering agents can radiate from a flare.

10. Periodical or quasi-periodical time variations in the frequency of stellar flares could be present as in the frequency of solar flares. 
(11) According to Cristaldi and Rodonò (1975) the Sun might be regarded as a low-activity flare star.

\subsubsection{Differences between Flares of UV Ceti-Type Stars and Solar Flares}

We also have differences between flares of UV Ceti-type stars and solar flares.

(1) Although stellar flares have been observed with rise time greater than $10 \mathrm{~min}$ and with total duration greater than $1 \mathrm{~h}$, generally the flares of UV Ceti-type stars have a lifetime of one order of magnitude, smaller than solar flares.

(2) While the total energy output in a stellar flare is of the same order of the undisturbed stellar continuum, the total energy output in a solar flare is about a millionth of the normal quiescent emission.

(3) Temperatures and probably densities of stellar flares are greater than temperatures of solar flares.

\subsubsection{The Problem of the Continuous Emission}

As is well known optical continuum emission from solar flares has been observed only in a few cases, mainly for very strong events known as white light flares. On the contrary, optical continuum emission during the flash phase is typical for all stellar flares. Certainly this difference is in part due to different detection conditions in the Sun and in the flare stars. On the other hand, as also pointed out by Haupt and Schlösser (1974), the ratio between the energy radiated in the optical continuum and total optical energy is much smaller for solar flares $(0.01)$ than for stellar flares $(0.5)$.

Two main mechanisms have been proposed for interpreting the optical continuum emission of solar flares.

According to Švestka (1970) in a limited volume of the flare region a strong impulsive process occurs shortly after the flare onset, accelerating protons and electrons up to the relativistic range for electrons and, in very strong events, up to the relativistic range of protons. Protons accelerated to energies above $20 \mathrm{MeV}$ penetrate down to the lowest chromospheric and upper photospheric layers and produce a heating of the atmosphere in the limited bombarded region which can be observed as a shortlived increase of the continuum in the optical spectral region.

According to Hudson (1971) decelerate electrons, diffusing out of the region where they produce the hard X-rays of the flash phase, could produce the optical continuum emission.

If we assume that one of these two mechanisms is responsible for the optical continuum emission of flare stars, we deduce that in the atmospheres of flare stars a larger number of particles must be accelerated.

Since during proton solar flares intensive proton and heavy element emission into interplanetary space is observed it is sound to explore if flare stars could be the source of the soft cosmic ray component (Lortet-Zuckermann, 1965).

Actually considering the rate of energy released during a stellar flare and adopting the factor, which has been determined empirically in the case of the Sun, for the conversion of flare energy to cosmic ray energy, Lovel (1974) showed that M type flare stars may be the major source of the galactic cosmic rays for energies from $10^{6}$ to 
$3 \times 10^{8} \mathrm{eV}$ and that $\mathrm{K}$ type stars may contribute to one fifth of the total cosmic-ray energy up to $10^{9} \mathrm{eV}$.

\section{Photospheric Stellar Activity}

\subsection{INTRODUCTION}

The hypothesis that non-uniform distribution of regions of constant brightness, coupled with rotation, could be responsible for stellar photometric variations, was proposed as early as the seventeenth century (see Ledoux and Walraven, 1958).

The hypothesis of variable superficial regions was introduced in the nineteenth century. Analogies with sunspots were called upon by Wolff and Secchi (1.c.).

According to Vardya (1970), the first plausible proof of the existence of stellar spots was found by Jaschek and Malaroda (1970) in the A2p star 73 Dra (HD 196502). These authors have found in 73 Dra, a star of effective temperature $\sim 9000 \mathrm{~K}$, lines of $\mathrm{CN}$ and $\mathrm{CH}$, corresponding to an effective temperature of $\sim 6600 \mathrm{~K}$. These molecular features, that seem variable, would indicate the presence of a cold spot.

As also pointed out by Piotrowsky et al. (1974) we could actually interpret any light curve assuming the existence of convenient spots on the photosphere of a rotating star. Therefore we must accept this assumption only if it either describes other known phenomena too or if photometric variations can not be implied in the frame of the known phenomenology.

Quite recently Friedemann and Gürtler (1975) calculated the photometric variations determined by the presence of one circular spot on the photosphere of a rotating star. Naturally a light curve depends (1) on the size of the spot, (2) on the temperature difference between the spot and the photosphere, (3) on the position of the spot on the stellar surface, $(4)$ on the orientation of the rotational axis in relation to the observer. Colour indices exhibit rather small changes $\left(\leq 0^{m} \cdot 05\right)$ especially for large temperature differences between the photosphere and the spot. Spots also lead to a shift of the star in the colour magnitude diagram.

Main classes of objects showing photospheric phenomena that could be and have indeed been interpreted in terms of photospheric activity are binary, magnetic and flare stars.

\subsection{Photospheric activity on Binary stars}

In 1930 Sitterly assumed that some distortion in the light curve of the binary system RS Canum Venaticorum could be due to photospheric spots. The same assumption was made by Kron (1947) and Struve (1952) for AR Lacertae and by Binnendijk (1970) for certain W Ursae Majoris systems. Quite recently it was taken up again and re-elaborated by Walter (1971) for SW Cygni and by Hall (1972) for RS Canum Venaticorum.

Due to the methodological reasons discussed, it might be more advisable to try to describe these distortions by taking into account gas-streams between components (see e.g. Catalano and Rodonò, 1974; Piotrowsky et al., 1974) required to explain other properties of these systems too. 
Notwithstanding this some authors still prefer a more elaborated modified spot assumption (Arnold and Hall, 1973; Hall, 1975; Mullan, 1975).

We notice that quite recently Weiler (1975a, 1975b) found for RS Canum Venaticorum and for other five binaries of the same type significant variations in the emission line intensities of the $\mathrm{H}$ and $\mathrm{K}$ line of $\mathrm{Ca}$ II and $\mathrm{H} \alpha$. At least for two systems maximum emission correlated with assumed spot activity was found.

\subsection{Photospheric activity ON MAgNetic STARS}

Very comprehensive and complete reviews are available on magnetic and related stars (e.g. Cameron, 1967; Pikelner and Khokhlova, 1971, 1972; Preston, 1971).

We recall here that all the observed magnetic fields, ranging from the limit of detectability of hundreds of gauss to $30 \mathrm{kG}$, seem to be variable with periods generally ranging from about 0.5 to 200 days.

Generally, stars with observable magnetic fields have peculiar spectra. Vice versa variable magnetic fields have been observed in all Ap stars having lines narrow enough for measurement of Zeeman splitting.

Also the other properties of these stars (brightness, colour, intensity of the spectral lines) generally show correlated periodic variations. Irregular variations of a short period are observed too.

Among the proposed models two are relevant for our discussion: the solar-cycle model and the oblique-rotator model.

The solar cycle model is based on the assumption that phenomena of the same type as those associated with the solar cycle, enhanced by orders of magnitude, could produce the observed variations. Although the variations observed in some stars could be actually interpreted with such a model (Blanco et al., 1972) some theoretical difficulty arises mainly connected with the high velocity required by the process of dissipation and reversal of magnetic fields naturally assumed to be superficial.

The oblique rotator model, having the axis of a static magnetic field inclined to the axis of rotation of the star, can describe fairly well the period vs line-width relation deduced by Deutsch for spectrum variables and the observed regular variations including the photometric one (Kodaira, 1973; Catalano, 1975). Notwithstanding this some difficulty arises for long period variables (Preston and Wolff, 1970; Catalano and Strazzulla, 1975). Moreover, active photospheric regions are required in this model too, in order to describe both short period irregular variations and the crossover effect.

Severny (1970), Borra and Landstreet (1973a, 1973b) and Boesgard (1974) opened the very promising possibility of observing weak stellar magnetic fields down to $15-20 \mathrm{G}$. Unfortunately results are still very scanty and no $\mathbf{M}$ dwarfs have been observed. It is interesting to note that some of the stars with weak magnetic fields have $\mathrm{Ca}$ II emission components.

\subsection{Photospheric activity ON flare stars}

Existence of slow, quasi periodic photometric variations of small amplitude (several hundredths of magnitude) in flare stars outside flare activity has been reported for many years. 
More recently Chugainov (1966) observed brightness variations in BY Dra (K6 V) with a period of 3.8 days and proposed to interpret these variations with the existence of a spot on the surface of a rotating star.

Following Chugainov's discovery, Krzeminsky and Kraft (1967) and Krzeminsky (1969) searched for similar brightness variations among six emission line objects (including BY Dra) and three non-emission line objects in the range of spectral types $\mathrm{dK} 7$ to dM 3.5. Four of the emission line objects showed variability in the range of 0.06-0.1 mag. Variability and period of BY Dra were confirmed; a period of 2.2 days was found for another of these four variable objects (FF And). The remaining emission line and the non-emission line stars showed no variability in excess of 0.02-0.04 mag.

Independently of the Krzeminsky and Kraft programme, the Catania group undertook in 1967, in the frame of its research project on stellar activity of the solar type, the study of luminosity variations between flares with the design of interpreting them assuming the presence of active regions (Godoli, 1967; Cristaldi et al., 1969; Godoli, 1968).

The observation of photometric variations of flare stars outside flare activity has been recently pursued by several authors (Andrews, 1968; Ferraz Mello and Torres, 1971; Torres et al., 1972; Chugainov, 1973, 1974; Vogt, 1973, 1975; Robinson and Kraft, 1974; Martins, 1975).

We notice that Robinson and Kraft (1974) observed Pleiades' and Hyades' dwarfs in the spectral-type range $\mathrm{K} 3 \mathrm{v}-\mathrm{M} 0 \mathrm{v}$ finding photometric variations outside flare activity in the Pleiades but not in the older Hyades. Actually Kraft and Greenstein (1969) found that the Pleiades contain more dMe stars than the Hyades.

Inconstancy of the amplitude of the photometric variations and of the mean brightness and colour was observed (Chugainov, 1973, 1974; Vogt, 1973). In BY Dra a steady increase in the mean light level is accompanied by a steady decrease in the amplitude of quiescent variations (Chugainov, 1973, 1974; Vogt, 1973).

Changes of period were observed by Chugainov $(1973,1974)$ although Vogt $(1973,1975)$ threw some doubt on this matter.

Evidence that flare activity may be almost the same independently of the periodical brightness variations was finally obtained by Chugainov (1974).

Evans (1971) attempted to interpret photometric variations of binary flare stors outside stellar activity, taking into account obscuring material temporarily located near Lagrangian points of the binary systems. But, stability considerations showed that the possible lifetime of circumstellar material near these points would be too short (Bopp and Evans, 1973).

Let us interpret photometric variations of flare stars outside flare activity as modulation by rotation of a spotted star (Kron, 1950a, 1950b, 1950c; Chugainov, 1966, 1973, 1974; Krzeminsky and Kraft, 1967; Krzeminsky, 1969; Torres et al., 1972; Bopp and Evans, 1973; Evans, 1973; Torres and Ferraz Mello, 1973; Vogt, 1973, 1975; Mullan, 1974).

Spots several hundredths of the stellar hemisphere large, extended to high latitude (up to $60^{\circ}$ ) with low effective temperature are needed. Estimations of the size of the spots can be made when the star belongs to a binary system. 
Inconstancy of the amplitude of the photometric variations and of the mean brightness could be due to variations of photospheric activity. For BY Dra Chugainov (1973) suggests an activity cycle of 8-9 yr.

Period changes could be due either, as on the Sun (Albrecht et al., 1969), to a migration in latitude of the spots in regime of differential rotation or to the presence of not constant, preferential longitudes.

The independence of flare activity from the periodical brightness variations, if confirmed, could point out that flares are not associated with photospheric activity. It would also rule out the possibility that brightness variations could be determined by bright active regions consisting of clumps of microflares.

Finally we note that equatorial rotational velocities of the order of $10-15 \mathrm{~km} \mathrm{~s}^{-1}$ would turn up. Such velocities, greater than expected for the lower end of the main sequence, may point, together with the presence of emission lines that we shall discuss further on, to the youth of the flare stars, although, as we have seen, some doubt exists on this topic.

If active regions really exist on flare stars strong magnetic fields associated with starspots ought to be present. The flare mechanism could consist in the release of energy by magnetic field collapse (Evans, 1973; Worden, 1974).

\subsection{CONCLUDING REMARKS ON PHOTOSPHFRIC STELLAR ACTIVITY}

Some of the assumptions on the existence $c$. star spots, that we have described, are extremely naïve.

We should always take into account not only spots but also other forms of activity that, on grounds of the solar analogy, ought to be present in stellar active regions. But for such an extension we need more observational details although some considerations have recently been made in this direction by Arnold and Hall (1973) for RS Canum Venaticorum-type binaries, by Mullan (1975) for W Ursae Majoris-type binaries and by Martins (1975) for flare stars.

\section{Chromospheric Stellar Activity}

\subsection{INTRODUCTION}

A section of the colloquium held in Münich in 1969 on the spectrum formation in stars with steady state extended atmospheres was dedicated to the chromospheres and coronae of stars (Groth and Wellman, 1970). Moreover an entire colloquium held at Goddard Space Flight Center in 1972 was dedicated to the problem of stellar chromospheres (Jordan and Avrett, 1973). For this reason we shall very briefly summarize here only the most important observational results and present the latest ones.

Arguments for the existence of stellar chromospheres and coronae are by now very convincing.

In the solar type situation we have a strong convection, kept by subphotospheric hydrogen ionization, which causes a host of waves. Among them the acoustic modes still appear to be the most important ones. As these waves propagate upward they steepen into shocks which dissipate into chromospheres and coronae. 
As an example of convective models we recall those by de Loore (1970) although it was pointed out at the Goddard Colloquium that the de Loore's models exaggerate the mechanical flux when convective zones are thinner than the mixing lengths. De Loore calculated photospheric models for 90 stars with effective temperatures ranging from $2500 \mathrm{~K}$ to $41600 \mathrm{~K}$ and acceleration of gravity ranging from 10 to $10^{5} \mathrm{~cm} \mathrm{~s}^{-2}$. He deduced that all the investigated stars contain unstable layers, including the hottest. Nevertheless, according to de Loore, only stars with the effective temperature of $8300 \mathrm{~K}$ or less contain layers where the convective energy transport is important. For main sequence stars, the largest fluxes would be generated in F2 III stars.

But, as pointed out by Thomas at the Münich Colloquium, we could have situations, other than the solar one, where chromospheres and coronae do exist as a consequence of mechanical instability different than acoustic waves. So we must not expect chromospheres and coronae only in stars of spectral class F0 and later ones (Lamers and de Loore, 1974).

Moreover the Catania group (Blanco et al., 1974) showed that for stars with $T_{\text {eff }}<5000 \mathrm{~K}$ the theoretical acoustic energy flux is inadequate, by two orders of magnitude for the coolest stars, to account for the observed chromospheric emission fluxes. New information by Blanco et al. (1975) on this matter will be reported in this section.

\subsection{STEllar CHROMOSPHERES}

Ca II resonance doublet emission ( $\mathrm{Ca} I \mathrm{H}$ and $\mathrm{K}$ at 3933.7 and $3968.5 \AA, 4 s^{2} S-$ $\left.4 p^{2} P^{\circ}\right)$ which is one of the best known indicators of stellar chromospheres was first reported by Eberhard and Ludendorff in Arcturus ( $\alpha$ Boo, K1 iII) (Eberhard and Schwarzschild, 1913). In their famous 1949 list Joy and Wilson reported 445 stars known to have $\mathrm{H}$ and $\mathrm{K}$ emissions (novae were excluded). The list indicated that, with few exceptions, calcium emission occurs only in stars of $G, K$ and $M$ types. Although supergiants, giants, subgiants are well represented in the Joy and Wilson list, the best represented are the dwarfs. Other stars with $\mathrm{H}$ and $\mathrm{K}$ emission components were found afterwards (Greenstein, 1952). A longer list was published by Bidelman (1954). In 1969 Warner (1969) added a list of 200 southern G, K and M stars with emission components. In 1970 Wilson and Woolley presented a list of calcium emission intensities of $\mathbf{3 2 5}$ main sequence late type stars.

The ultraviolet magnesium counterpart of the $\mathrm{Ca}$ II resonance doublet emission (Mg II resonance doublet at 2795.5 and $2802.7 \AA, 3 s^{2} S-3 p^{2} P^{\circ}$ ), was first reported in the solar spectrum by Durant $e t$ al. in 1949. In late type stars it was reported by Doherty (1971, 1972), Gurzadian (1972), Kondo (1972), Kondo et al. (1972), Linsky and Basri (1974), Moos et al. (1974), Kondo et al. (1975); in intermediate type stars by Kondo (1972), Dupree (1974); in a wide range of spectral types by Oganesyan (1974).

Chromospheric Ly- $\alpha$ emission was observed from Arcturus by Rottman et al. (1971), Moss and Rottman (1972), Moss et al. (1974) and from an intermediate type star by Dupree (1974). O I $\lambda 1304$ was observed from Arcturus by Moss and Rottman (1972). O II $\lambda 1334.5$ was observed in an intermediate type star by Dupree 
(1974). Other ultraviolet lines were observed in the spectrum of Procyon ( $\alpha$ CMi F5 Iv) (Evans et al., 1975).

Rosendhal (1973) found changes in the strength or structure of the $\mathrm{H} \alpha$ as well as in the strength of other prominent lines in the red region of the spectrum of 13 early type supergiants among the 20 examined. This behaviour could be due to possible coupling between mass loss and turbulence.

Linsky (1973) reported that in the Sun the profile of the Ca II triplet line at $\lambda 8498 \AA$ shows a definite double reversal in the weak and slightly stronger plages. Therefore Linsky suggested that the $8498 \AA$ line could be a very sensitive indicator of the activity of stellar chromospheres. Anderson (1973) obtained photoelectric profiles of this line for $\mathbf{3 0}$ late-type stars but at the resolution and precision of his study none of the stars observed show any evidence of emission although several have very strong $\mathrm{K}$ line emission.

Vaughan and Zirin (1968) pointed out that the $\lambda 10830 \AA$ line of neutral helium is a better indicator for the existence of stellar chromospheres than $\mathrm{Ca}$ II lines: on the Fraunhofer solar spectrum, indeed, the $\lambda 10830 \AA$ line is the only one in the range from $3000 \AA$ to $11000 \AA$ that originates solely in the chromosphere; moreover $\mathrm{H}$ and $\mathrm{K}$ lines of $\mathrm{Ca} \mathrm{II}$ and $\mathrm{H} \alpha$ line are excited in a wide range of temperature and may show either enhanced emission or absorption depending upon physical conditions. These authors have found in a wide research on 86 stars the $\lambda 10830 \AA$ line of neutral helium in absorption in a substantial number of $G$ and early $K$ stars and in emission in five stars. According to Vaughan and Zirin the He line would probably not be present, at least with appreciable strength in either $F$ or $M$ stars. However we notice that, as far as $M$ stars are concerned, only supergiants, bright giants and giants have been observed.

As early as $\mathbf{5 0}$ years ago, Stratton realized that $\mathrm{Ca}$ II resonance doublet emissions were correlated with the luminosity of the late type stars. After many years Wilson $(1954,1959,1967,1970)$ and Wilson-Bappu (1957) gave the relationship between the Ca II emission line width and the absolute visual magnitude. Since this relationship is valid for over a range of more than $15 \mathrm{mag}$ and it does apply to the Sun too, we can expect that the mechanism which produces the line widths is the same for all the stars including the Sun.

Further analysis of this effect has been carried out by Reimers (1973).

A relationship between $\mathrm{H}$ and $\mathrm{K}$ absorption lines and luminosities was also reported in the range of spectral types from about F5 to K2 (Lutz et al., 1973).

Mg II resonance doublet emissions also follow the Wilson Bappu relationship (Kondo, 1972; Kondo et al., 1975). According to Kondo et al. (1972) the widths of the $\mathrm{Mg}$ II emission lines are between two and three times greater than the widths of $\mathrm{Ca}$ II emission lines for a given star.

A relationship between $\mathrm{Ca}$ II and $\mathrm{Mg}$ II resonance doublet absorption and luminosity was recently investigated by Ayres et al. (1975).

Considering that, on the Sun, the absorption structures at the edges of the core of $\mathrm{H} \alpha$ line correspond to the emission structures of $\mathrm{K}_{2}$ (McMath et al., 1956), Kraft $e t$ al. (1964) searched for and found in the spectra of late type stars an increase in the width of the $\mathrm{H} \alpha$ core in absorption with increasing luminosities. Further measurements were made by LoPresto (1971) (McMath solar telescope) and by Caplan (1973). 
Bonsack and Culver (1966) found such a behaviour also for weak absorption lines formed in the deeper atmosphere of $\mathrm{K}$ type stars. According to these authors, the profiles of the weak lines in $\mathrm{K}$ stars are dominated by the motions of large elements of gas. Therefore the velocities of these elements must be correlated with stellar luminosity in the same way as the phenomena at higher levels which determine the widths of the $\mathrm{K}$ line emission and $\mathrm{H} \alpha$ absorption.

According to Anderson (1973) the central intensity as well as the equivalent width of the Ca II triplet line at $\lambda 8498 \AA$ show no evidence of the Wilson Bappu effect in thirty late-type stars observed.

The existence of chromospheres of the UV Ceti-type stars has been suspected since the first spectra of these stars were obtained outside flare activity.

Discussing their list, Joy and Wilson pointed out that the strongest $\mathrm{K}$ emissions appear in $\mathrm{T}$ Tauri stars and in the dMe dwarfs of extremely low luminosity.

In 1960 Wilson (1961) obtained at Palomar a sprectrogram of EV Lac covering the photographic region with a dispersion of $9 \AA \mathrm{mm}^{-1}$. He observed emission lines of $\mathrm{H}, \mathrm{He}_{\mathrm{I}}, \mathrm{Si}$ I, $\mathrm{Ca}$ I, $\mathrm{Fe}_{\mathrm{I}}, \mathrm{Ca}$ II. Since the excitation requirements for these lines are different by one order of magnitude, Wilson suggested a stratification of chromospheric layers. From the sharpness of the metallic lines and the lack of differential velocities he assumed that thermal motion is probably the principal source of broadening for the hydrogen lines. With this assumption he deduced a temperature of about $14000 \mathrm{~K}$ for the chromospheric zone giving rise to the hydrogen emission.

On the other hand, Gershberg (1970) found that the number of hydrogen atoms per unit area of the chromosphere in UV Ceti-type stars is greater by a factor of 3-50 than the corresponding number for solar chromosphere.

Jennings and Dyck (1972) and Jennings (1973) showed that $\mathrm{H}$ and $\mathrm{K}$ reversals and other heavy-element emission lines, especially those of ionized species, tend to vanish in stars which exhibit intrinsic polarization and infrared excess. The infrared excess, measured by the 0 -L colour (the magnitude at $11 \mu$ minus the magnitude at $3.5 \mu$ ) has been interpreted by thermal reradiation from circumstellar mineral grains. Since the infrared excesss is strongly correlated with polarization, scattering by particle must be responsible for observed intrinsic polarization. According to Jennings and Dyck the disappearance of emission lines implies that grains are responsible for the weakening of the chromospheres through a mechanism involving thermodynamic or indirect dynamic effects or a combination of the two.

\subsection{Chromospheric STEllar ACTIVItY}

According to some authors (e.g. Unsöld, 1964) we could consider intensities of $H$ and $\mathrm{K}$ emission components not only as chromospheric indicator $S$ but also as chromospheric activity indicators.

We must point out that solar $\mathrm{H}$ and $\mathrm{K}$ emission components, in integrated sunlight, are below the limit of detectability at those dispersions generally used in the observations of stellar $\mathrm{H}$ and $\mathrm{K}$ emissions. We can therefore deduce that solar type stars with observed $\mathrm{H}$ and $\mathrm{K}$ emissions must have more active chromospheres than the Sun. 
$\mathrm{Ca} \mathrm{II} \mathrm{H}$ and $\mathrm{K}$ emission component intensities (and hence the general degree of the chromospheric activity) and the angular velocity of a main sequence star, decay as the inverse square root of the age (Wilson, 1963; Wilson and Skumanich, 1964; Skumanich, 1972; Boesgaard and Hagen, 1974). Assuming that the factor which determines the intensity of $\mathrm{H}$ and $\mathrm{K}$ emissions in the chromosphere of a star is simply the average magnetic field strength over its surface, one can predict that the magnetic field strength also gradually decays as the star ages and moreover that angular velocity must be greater when the magnetic field is stronger.

Since late type giants possess a more intense chromosphere than their counterparts on the main sequence it would be necessary to explain how the magnetic field could be reactivated when the star begins to evolve upwards to the giant branch in the H-R diagram (Matsushima, 1974). According to Matsushima (1974) a decrease of metal content could produce an increase of the surface convective flux.

According to the Catania group (Blanco et al., 1974) the Sun as a whole does not fit in with the relations between $\mathrm{H}$ and $\mathrm{K}$ emission component intensities and age unless only active regions are considered. Therefore we must consider stellar $\mathrm{H}$ and $\mathrm{K}$ emission components as indicators of chromospheric stellar activity rather than, simply, as indicators of stellar chromospheres.

Apart from active regions, we know nowadays that the brightness of knots of the solar chromospheric network at the centre of the Sun and at the northern and southern poles changes with the phase of the solar cycle (Tsap and Labe, 1973).

Therefore, solar analogies would suggest that it is better to search for chromospheric stellar activity by analysing stars with variable emission components.

Many stars, in which the $\mathrm{H}$ and $\mathrm{K}$ emission components are probably variable, are already indicated in the Joy and Wilson list.

For a period of about 5 years (from 1950 to 1954) Popper (1956) found no definite intensity change in $H$ and $K$ emissions of five main sequence $K$ and $M$ stars.

As far as I know, definite variation in the intensity of $\mathrm{H}$ and $\mathrm{K}$ emissions was first observed in 1962 by Kandel in HD 119850 (spectral type dM 2.5) (Kandel, 1962, 1966). In 1963 Griffin discovered variations in Arcturus (K1 III). Deutsch (1967) found large changes in the profiles of $\mathrm{Ca}$ II $\mathrm{H}$ and $\mathrm{K}$ in two giants ( $\alpha$ Tauri, $\mathrm{K} 5 \mathrm{III}$ and $\gamma$ Aquilae K3 II) and smaller changes in most other $\mathrm{K}$ giants examined. Large profile changes have been found in $\alpha$ Tauri only after an interval of 100 days or more. The changes are not regular, but they tend to reoccur after about 1000 days, probably the rotational period of the star.

Nine stars in the spectral range G2-M0, observed by Liller (1968) in the period September 1964 - August 1965, showed, almost continuously, small random variations of the $\mathrm{K}$ line emission component in both intensity and shape. The stars $\alpha$ Tauri and, possibly, $\lambda$ Andromedae exhibit greater fluctuations sometimes of flare-like nature with a recovery time of the order of half an hour. No evidence of cyclic variations was detected.

Boesgaard (1969) found fairly strong $\mathrm{H}$ and $\mathrm{K}$ emission components in a M3 S star in which no emission had been observed for many years.

Vaughan and Zirin (1968) observed temporal changes of the neutral helium $\lambda$ 10830 emission line in two of the five stars showing this line in emission. 
Since 1965 photoelectric flux measurements at the centre of stellar $\mathrm{H}$ and $\mathrm{K}$ lines have been systematically performed at Mount Wilson with the main goal of collecting data which may ultimately lead to the detection and study of stellar analogues of the solar cycle (Wilson, 1968). According to Wilson (1968) no undoubted variations were found during the first year observations.

Soon afterwards, Wilson (1969) announced that flux variations in both components of 61 Cygni had been found during the period June-December 1967.

Nowadays we know that for several of the stars observed by Wilson it is likely that a cycle has nearly been completed. Up to now none of these stars have been reported to begin a new cycle (Hale Observatories, Annual Report 1973-74). New information by Wilson (1976) will be reported in this section.

Active chromospheres in eclipsing binary stars were observed for a long time (Wright, 1973).

Chromospheric activity on flare stars was observed by Popper in 1953 on BY Dra $(\mathrm{K} 6 \mathrm{v})$ when this star was not yet known to be a flare star. On a spectrogram obtained on 1953 July 21,37 UT he observed hydrogen emission lines many times stronger than on the other plates. $\mathrm{H}$ and $\mathrm{K}$ lines were probably also strengthened.

Recently Bopp (1974a, b) discovered variations of up to a factor of three in the equivalent widths of the emission lines of seven flare stars (among nine observed) at a time when no detectable flare was photometrically in progress. The time scale of this variation is of the order of one day or less. As Bopp (1974a) also points out, such a time scale shows that quiescent chromospheric emission must be localized: a variable emission produced by a uniform chromosphere would in fact imply a corresponding variation of the convective transport over the entire stellar surface. According to Bopp the observations are best explained if one assumes that active regions are present on a rotating star with the rotation axis inclined at a considerable angle to the plane of the sky in such a way that the active regions, although partially visible in all their lifetime are observed with varying degrees of foreshortening and limb darkening. Rotational velocities from five to twenty times the solar rotational velocity are found, as obtained from photospheric activity features.

\section{Coronal Stellar Activity}

\subsection{INTRODUCTION}

Stellar coronal parameters have been computed by many authors under the main assumption that the mechanical energy flux from convective layers is responsible for the heating of the corona (de Jager and Neven, 1961; Kuperus, 1965; Bierman, 1969; Nariai, 1969; de Loore, 1970; de Jager and de Loore, 1971). According to coronal models constructed by de Loore (1970) for the Sun and for stars with effective temperatures between $5350 \mathrm{~K}$ and $8320 \mathrm{~K}$, the stars with $T_{\text {eff }}=7130 \mathrm{~K}$ and $\log g=4$ possess the hottest and densest coronae with a computed temperature of $3.7 \times 10^{6} \mathrm{~K}$ and $\log N_{e}=10.4$.

Expected stellar radio fluxes (Weimann and Chapman, 1965; Oster, 1971) and X fluxes (Bierman, 1969; de Jager, 1971; de Loore and de Jager, 1970; Landini and Monsignori Fossi, 1973) have been computed. 
A radio candidate was

Betelgeuse ( $\alpha$ Ori, Me I);

$\mathrm{X}$ candidates were

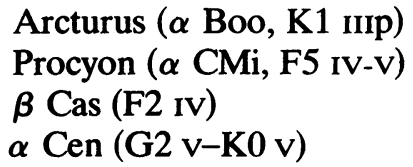

Since the gravitational potential of giants and particularly of supergiants is smaller than that of main sequence stars, one could expect no coronas of solar type in these stars (Bierman and Lust, 1960) or perhaps, better, since a large supply of acoustic energy is available, a stellar wind much more powerful than the solar wind. Actually, attempts were made in interpreting mass loss in red giants by a stellar wind mechanism (Weymann and Chapman, 1965; Fusi Pecci and Renzini, 1975).

\subsection{Stellar CORONAE}

While observing chromospheric Ly- $\alpha$ emission from $\beta$ Gem (K0 III) with the 'Copernicus' Satellite, McClintock et al. (1974) detected an emission line at $1218.4 \pm 0.2 \AA$ identified with the $2 s^{2}{ }^{1} S_{0}-2 p{ }^{3} P_{1}$ intercombination line of $\mathrm{O} v$. This line was first detected and identified in the solar limb spectra obtained by Burton et al. in 1965 (Burton et al., 1967). This identification was subsequently confirmed by laboratory measurements by Edlén et al. (1969) and by new observations by Burton and Ridgeley (1970). In the solar spectrum this line has a strength smaller than 0.03 that of Ly- $\alpha$. In $\beta$ Gem it has a strength $\simeq 0.3$ that of Ly- $\alpha$. According to McClintock et al. (1974), calculations indicate that in $\beta$ Gem the line is formed in a corona at temperatures near $260000 \mathrm{~K}$ rather than in a transition region as in the solar case. Other high-excitation lines are being searched for in $\beta$ Gem. The $\mathrm{Ov}$ line is not observed in Aldebaran ( $\alpha$ Tau, K5 III), Arcturus ( $\alpha$ Boo, K1 III), or $\varepsilon$ Eri (K2 v) which have been similarly studied by the Copernicus satellite.

If stellar coronae do exist, it is to be expected that the scattering of light by free electrons would cause the apparent angular distribution of light across the star to depend upon the plane of polarization. Hanbury Brown et al. (1974) made observations of the hot supergiant $\beta$ Ori (B8 ra) with the stellar intensity interferometer at Narrabri using linearly polarized light. The angular diameter was measured at two base lines with light polarized parallel and perpendicular to the base line and the results were used to derive two independent measures of the angular size of the star. No significant change of angular diameter with polarization was observed. From computation of the effects of the expected polarization the authors concluded that to obtain positive results it would be necessary to improve the sensitivity of the measurements by a factor in a range of 10 to 100 .

\subsection{Coronal stellar activity}

Significant flux density of $0.11 \pm 0.03$ flux units from Betelgeuse was detected at a wavelength of $1.9 \mathrm{~cm}$ by Kellermann and Pauliny-Toth in 1966, February 21 at the 
National Radio Astronomical Observatory. No significant flux was found in the next or in ten other nights in February and March. Low (1965) had found strong infrared radiation from this star at $10 \mu$, variable in intensity, but unfortunately no infrared measurements are available during the period of radio measurements.

Probable significant fluxes from Betelgeuse (M2 Ib) and $\pi$ Aurigae (M3 II) were detected at a $\lambda$ of $2.85 \mathrm{~cm}$ by Seaquist (1967) at the Algonquin Radio Observatory on 1966 October 30 and August 13 respectively. No significant flux was found from $\delta$ Orionis (O 9.5 II) and $\psi$ Aurigae (M0 Iab) on 1960 October 30.

Significant flux density of $0.005 \pm 0.001$ flux units from Antares ( $\alpha$ Scorpii) was detected at a wavelength of $11.1 \mathrm{~cm}$ by Wade and Hjellming (1971) at the NRAO on 1970, June 4, and November 12. No significant flux was detected at $3.7 \mathrm{~cm}$. According to Oster (1971) what Wade and Hjellming observed may have been a stellar type IV burst or a series of them.

Further observations (Hjellming and Wade 1971a, b) showed that Antares' radio source is variable, detectable at both 3.7 and $11.1 \mathrm{~cm}$ and associated with the B3 v companion to the red supergiant M1 $\mathrm{Ib}$. According to Hjellming and Wade this radio emission could be related either to the infall of matter from the red supergiant or to particles' acceleration and ejection from the surface of the B star itself. This second possibility is the interesting one in our framework.

Many other objects of this kind have been discovered in the last three years.

Radioemission was found from a Bep star by Braes et al (1972). Also this star has an infrared excess, probably due to ejected circumstellar material (Geisel, 1970). Other objects of this kind have been discovered.

Catura et al. $(1974,1975)$ recently detected X-ray emission in the range from 0.2 to $1.6 \mathrm{keV}$ from an area of the sky which contains the binary star system Capella ( $\alpha$ Aurigae). Capella is a spectroscopic binary at 15 pc consisting of an F8-G0 giant of $2.9 \mathrm{M}$ and a G5 giant of $3.0 \mathrm{M}$. The system rotates in a nearly circular orbit with a period of 104.023 days. The separation between the stars is of about $1 \mathrm{AU}$. No radio emission from the Capella system has been reported.

The detector was pointed at Capella in 1974, April 5 for a 1.2 period. The number of counts obtained when Capella was within the field of view was about 10 times the average counting rate. The authors are of the opinion that it is very improbable that this signal is a random fluctuation in the background counting rate. The fact that the spectrum shows no indication of a turnover at $0.25 \mathrm{keV}$ from $\mathrm{X}$-ray absorption by interstellar matter, is consistent with a nearby source. If this identification is correct, an X-luminosity of Capella during the observation comes out ranging from $10^{31}$ to $10^{34} \mathrm{erg} \mathrm{s}^{-1}$.

Fitting the data with a function describing thermal bremsstrahlung the authors found temperatures ranging from 5 to $15 \times 10^{6} \mathrm{~K}$.

This source was not observed during a previous survey of the same region in 1972. The authors recall other soft X-ray sources, identified with nearby stellar objects, which other observations of comparable or better sensitivity have failed to detect. According to the authors one must conclude that either many spurious observations have been reported, or these sources of soft X-ray emission are strongly variable in time.

Comparison of these events with those observed on YZCMi and UV Ceti is stimulating. 


\section{Concluding Remarks}

I should like to conclude this review on the observational aspects of stellar activity of the solar type with some remarks.

Although, as we have seen, information on Ap stars can be extremely stimulating in our framework, it is advisable to look for objects with much weaker magnetic fields. The recently opened possibility of observing stellar weak magnetic fields must therefore be pursued.

As we have seen, there is a lot of information that could be collocated in our framework. Notwithstanding this, we have not yet ascertained the existence of stellar activity cycles and we are just beginning to understand some associations among different known manifestations of stellar activity.

What we absolutely need to substantially improve the situation is an international cooperative effort that must exploit all the different available techniques and must be concentrated for a long time on a few objects of different types. We cannot hope the stars will help us in understanding the Sun unless we observe them as we observe the Sun.

\section{Acknowledgements}

It is a pleasure to thank F. Catalano, S. Catalano and M. Rodonò for helpful discussions and S. Motta and M. Rodonò for reading the manuscript.

\section{References}

Albrecht, R., Maitzen, H. M., and Rakos, K. D.: 1969, Astron. Astrophys. 3, 236.

Ambartsumian, V. A. and Mirzoyan, L. V.: 1971, IAU Colloq. 15, 98.

Anderson, C. M.: 1973, Bull. Am. Astron. Soc. 5, 413.

Andrews, A. D.: 1968, IAU Comm. 27, Inf. Bull. Var. Stars 273.

Arnold, C. N. and Hall, D. S.: 1973, IAU Comm. 27, Inf. Bull. Var. Stars 843.

Ayres, T. R., Linsky, J. L., and Shine, R. A.: 1975, Astrophys. J. 195, L121.

Babcock, H. W.: 1968, Ann. Rep. Director Mt. Wilson Palomar Obs. 1966-67, p. 262.

Babcock, H. W.: 1974, Ann. Rep. Director Hale Obs. 1973-74, p. 136.

Bappu, M. K. V.: 1967, Kodaikanal Obs. Repr. No. 37.

Bateson, F. M.: 1971, Southern Stars 24, 23.

Batten, A. H.: 1973, Binary and Multiple Systems of Stars, Pergamon Press, Oxford.

Bidelman, P.: 1954, Astrophys. J. Suppl. 1, 175.

Biermann, L. and Lüst, R.: 1960, Stars Stellar Systems 6, 260.

Biermann, L.: 1969, Proc. Roy. Soc. London A313, 357.

Binnendijk, L.: 1969, Astron. J. 74, 1031.

Binnendijk, L.: 1970, Vistas Astron. 12, 217.

Blanco, C., Catalano, F. A., Godoli, G., and Vaccari, S.: 1972, Mem. Soc. Astron. Ital. 43, 655.

Blanco, C., Catalano, S., Marilli, E., and Rodonò, M.: 1974, Astron. Astrophys. 33, 257.

Blanco, C., Catalano, S., and Marilli, E.: 1976, this volume, p. 473.

Boesgaard, A. M.: 1969, Publ. Astron. Soc. Pacific 81, 283.

Boesgaard, A. M.: 1974, Astrophys. J. 188, 567.

Boesgaard, A. M. and Hagen, W.: 1974, Astrophys. J. 189, 85.

Bonsack, W. K. and Culver, R. B.: 1966, Astrophys. J. 145, 767.

Bopp, B. W. and Evans, D. S.: 1973, Monthly Notices Roy. Astron. Soc. 164, 343.

Bopp, B. W. and Moffett, T. J.: 1973, Astrophys. J. 185, 239. 
Bopp, B. W.: 1974a, Monthly Notices Roy. Astron. Soc. 166, 79.

Bopp, B. W.: 1974b, Monthly Notices Roy. Astron. Soc. 168, 255.

Bopp, R. W. and Feckel, F. Jr.: 1974, Publ. Astron. Soc. Pacific 86, 978.

Borra, E. F. and Landstreet, J. D.: 1973a, Astrophys. J. 185, L139.

Borra, E. F. and Landstreet, J. D.: 1973b, Astrophys. J. 185, L145.

Braes, L. L. E., Habing, H. J., and Schoenmaker, A. A.: 1972, Nature 240, 230.

Bray, R. J. and Loughhead, R. E.: 1964, Sunspots, Chapman and Hall Ltd., London, p. 282.

Bumba, V. and Howard, R.: 1965, Astrophys. J. 141, 1502.

Bumba, V., Howard, R., and Smith, S. F.: 1967, in R. C. Cameron (ed.) Magnetic and Related Stars, Mono Book Corporation, Baltimore, p. 131.

Bumba, V. and Rủžicková-Topolová, B.: 1967, Solar Phys. 1, 216.

Burton, W. M., Ridgeley, A., and Wilson, R.: 1967, Monthly Notices Roy. Astron. Soc. 135, 207.

Burton, W. M. and Ridgeley, A.: 1970, Solar Phys. 14, 3.

Cameron, R. C.: 1967, The Magnetic and Related Stars, Mono Book Corporation, Baltimore.

Caplan, J. G.: 1973, Astron. Astrophys. 28, 213.

Catalano, F. A.: 1975, Mem. Soc. Roy. Sci. Liège VI Series 7, 117.

Catalano, F. A. and Strazzulla, G.: 1975, IAU Colloq. 32, (to be published), preprint.

Catalano, S. and Rodonò, M.: 1974, Publ. Astron. Soc. Pacific 86, 390.

Catura, R. C., Acton, L. W., and Johnson, H. M.: 1974, Bull. Am. Astron. Soc. 6, 445.

Catura, R. C., Acton, L. W., and Johnson, H. M.: 1975, Astrophys. J. 196, L47.

Chugainov, P. F.: 1966, Comm. 27 IAU, Inf. Bull. Var. Stars 122.

Chugainov, P. F.: 1973, Izv. Krymsk. Astrofiz. Obs. 48, 3.

Chugainov, P. F.: 1974, Izv. Krymsk. Astrofiz. Obs. 52, 3.

Crannell, C. J., McClintock, J. E., and Moffett, T. J.: 1974, Nature 252, 659.

Cristaldi, S., Godoli, G., Narbone, M., and Rodonò, M.: 1969, in 'Non-Periodic Phenomena in Variable Stars', IAU Colloq., Academic Press, Budapest, p. 149.

Cristaldi, S. and Rodonò, M.: 1975, IAU Symp. 67, 75.

Cristaldi, S. and Rodonò, M.: 1976, Astron. Astrophys. 48, 165.

Deutsch, A. J.: 1967, Publ. Astron. Soc. Pacific 79, 431.

Doherty, L. R.: 1971, Phil. Trans. Roy. Soc. London A270, 189.

Doherty, L. R.: 1972, Astrophys. J. 178, 495.

Dupree, A. K.: 1974, Bull. Am. Astron. Soc. 6, 446.

Durand, E., Oberly, J. J., and Tousey, R.: 1949, Astrophys. J. 109, 1.

Durney, B. R. and Stenflo, J. O.: 1972, Astrophys. Space Sci. 15, 307.

Eberhard, G. and Schwarzschild, K.: 1913, Astrophys. J. 38, 292.

Edlén, B., Palenius, H. P., Bockasten, K., Hallin, R., and Bromander, J.: 1969, Solar Phys. 9, 432.

Evans, D. S.: 1971, Monthly Notices Roy. Astron. Soc. 154, 329.

Evans, D. S.: 1973, Bull. Am. Astron. Soc. 5, 400.

Evans, R. G., Jordan, C., and Wilson, R.: 1975, Nature 253, 612.

Ferraz Mello, S. and Torres, C. A. O.: 1971, IAU Comm. 27, Inf. Bull. Var. Stars 577.

Friedemann, C. and Gürtler, J.: 1975, Astron. Nachr. 296, 125.

Fusi Pecci, A. and Renzini, A.: 1975, Astron. Astrophys. 39, 413.

Geisel, S. L.: 1970, Astrophys. J. 161, L105.

Gerola, H., Linsky, J. L., Shine, R., McClintock, W., Henry, R. C., and Moos, H. W.: 1974, Astrophys. J. 193, L107.

Gershberg, R. E.: 1967, Astrophysics 3, 64.

Gershberg, R. E., Neshpor, Y. I., and Chugainov, P. F.: 1969, Izv. Krymsk. Astrofiz. Obs. 39, 140.

Gershberg, R. E.: 1970, Astrophysics 6, 92.

Gershberg, R. E.: 1971, Flares of Red Dwarf Stars (transl. from the Russian by D. J. Mullan), Armagh Obs., N. Ireland.

Gershberg, R. E. and Pikelner, S. B.: 1972, Comments Astrophys. Space Sci. 4, 113.

Gershberg, R. E.: 1975, IAU Symp. 67, 47.

Gershberg, R. E. and Lund, L.: 1975, Proc. III European Astron. Meeting.

Giglas, H. L., Burnham, R. Jr., and Thomas, N. G.: 1967, Lowell Obs. Bull. No. 138.

Gliese, W.: 1969, Veroeffentl. Astron. Rechen. Inst. Heidelberg No. 22.

Godoli, G.: 1967, Oss. astrofis. Catania Pubbl. No. 115.

Godoli, G.: 1968, in Y. Ohman (ed.) Mass Motions in Solar Flares and Related Phenomena, Wiley Interscience Division, New York, p. 211.

Greenstein, J. L.: 1952, Publ. Astron. Soc. Pacific 64, 71.

Greenstein, J. L. and Arp, H.: 1969, Astrophys. Letters 3, 149.

Griffin, R. F.: 1963, Observatory 83, 255. 
Grindlay, J. E.: 1970, Astrophys. J. 162, 187.

Grindlay, J.: 1975, Private communication.

Grindlay, J. and Heise, J.: 1975, 'Observation of X Ray from Flare Stars with ANS' (preprint).

Groth, H. G. and Wellmann, P.: 1970, Spectrum Formation in Stars with Steady State Extended Atmospheres, Nat. Bureau Standard, Washington, Special Publ. 332.

Gurzadian, G. A.: 1965, Astrophysics 1, 170.

Gurzadian, G. A.: 1966a, Astrophysics 2, 109.

Gurzadian, G. A.: 1966b, Dokl. Akad. Nauk SSSR 166, 53.

Gurzadian, G. A.: 1971, Astron. Astrophys. 13, 348.

Gurzadian, G. A.: 1972, Astron. Astrophys. 20, 145.

Gurzadian, G. A.: 1972, Sky Telesc. 43, 350.

Hall, D. S.: 1972, Publ. Astron. Soc. Pacific 84, 323.

Hall, D. S.: 1975, Preprint.

Hanbury Brown, R., Davis, J., and Allen, L. R.: 1974, Monthly Notices Roy. Astron. Soc. $168,93$.

Harrington, R. S., Dahn, C. C., and Guetter, H. H.: 1974, Astrophys. J. 194, L87.

Haupt, W. and Schlosser, W.: 1974, Astron. Astrophys. 37, 219.

Heise, J., Brinkman, A. C., Schrijver, J., Mewe, R., Gronenschild, E., Den Boggende, A., and Grindley, J.: 1975, Astrophys. J. 202, 73.

Hjellming, R. M. and Wade, C. M.: 1971a, Astrophys. J. 168, L115.

Hjellming, R. M. and Wade, C. M.: 1971b, Science 173, 1087.

Howard, R.: 1972, Science 177, 1157.

Hudson, H. S.: 1972, Solar Phys. 24, 414.

Jager, C. de and Neven, L.: 1961, Mem. Soc. Roy. Sci. Liège, V. Serie, 4, 552.

Jager, C. de: 1971, Phil. Trans. Roy. Soc. London A270, 175.

Jager, C. de and Loore, C. de.: 1971, Astrophys. Space Sci: 11, 284.

Jaschek, M. and Malaroda, S.: 1970, Nature 225, 246.

Jennings, M. C. and Dyck, H. M.: 1972, Astrophys. J. 177, 427.

Jennings, M. C.: 1973, Astrophys. J. 185, 197.

Jerzykiewicz, M. and Serkowski, K.: 1966, Lowell Obs. Bull. 6, 295.

Johnson, H. L. and Iriarte, B.: 1959, Lowell Obs. Bull. 4, 99.

Jordan, S. D. and Avrett, E. H.: 1973, Stellar Chromospheres, NASA, Washington, SP-317.

Joy, A. H. and Wilson, R. E.: 1949, Astrophys. J. 109, 231.

Kahn, F. D.: 1974, Nature, 250, 125.

Kandel, R.: 1962. Compt. Rend. Acad. Sci. Paris 255, 1575.

Kandel, R.: 1966, in M. Hack (ed.), Colloquium on Late Type Stars, Oss. Astron., Trieste, p. 146.

Kellermann, K. I. and Pauliny Toth, I. I. K.: 1966, Astrophys. J. 145, 953.

Klimishin, I. A.: 1970, Tsirk. ShA. O. 6, 13.

Kodaira, K.: 1973, Astron. Astrophys. 26, 385.

Kondo, Y.: 1972, Astrophys. J. 171, 605.

Kondo, Y., Giuli, R. T., Modisette, J. L. and Rydgren, A. E.: 1972, Astrophys. J. 176, 153.

Kondo, Y., Morgan, T. H., and Modisette, J. L.: 1975, Astrophys. J. 196, L125.

Korovyakovskaya, A. A.: 1972, Astrophysics, 8, 148.

Kraft, R. P., Preston, G. W., and Wolff, S. C.: 1964, Astrophys. J. 140, 235.

Kraft, R. P. and Greenstein, J. L.: 1969, in S. Kumar (ed.), Low Luminosity Stars, Gordon and Breach, London, p. 65.

Kron, G. E.: 1947, Publ. Astron. Soc. Pacific 59, 261.

Kron, G. E.: 1950a, Publ. Astron. Soc. Pacific 62, 141.

Kron, G. E.: 1950b, Astron. J., 55, 69.

Kron, G. E.: 1950c, Astron. Soc. Pacific Leaflet 6, No. 257.

Krzeminsky, W. and Kraft, R. P.: 1967, Astron. J. 72, 307.

Krzeminsky, W.: 1969, in S. Kumar (ed.), Low Luminosity Stars, Gordon and Breach, London, p. 57.

Kunkel, W. E.: 1967, Astron. J. 72, 810.

Kunkel, W. E.: 1970, Publ. Astron. Soc. Pacific 82, 1341.

Kunkel, W. E.: 1973, Astrophys. J. Suppl. 25, 1.

Kunkel, W. E.: 1975, IAU Symp. 67, 15.

Kunkel, W. E. and Zarate, N.: 1975, Preprint.

Kuperus, M.: 1965, Rech. Astron. Obs. Utrecht 17, No. 1.

Lamers, H. J. G. L. M. and Loore, C. de: 1974, Preprint.

Landini, M. and Monsignori Fossi, B. C.: 1973, Astron. Astrophys. 25, 9.

Ledoux, P. and Walraven, Th.: 1958, Handbuch der Physik 51, 353.

Liller, W.: 1968, Astrophys. J. 151, 589. 
Linsky, J. L.: 1973, in S. D. Jordan and E. H. Avrett (eds.), Stellar Chromospheres, NASA, Washington, SP-317, p. 48.

Linsky, J. L. and Basri, G.: 1974, Bull. Am. Astron. Soc. 6, 458.

Lo Presto, J. C.: 1971, Publ. Astron. Soc. Pacific 83, 674.

Loore, C. de: 1970, Astrophys. Space Sci. 6, 60.

Loore, C. de and Jager, C. de: 1970, IAU Symp. 37, 238.

Lortet-Zuckermann, M. C.: 1965, Kleine Veroeffentl. Remeis Sternw. Bamberg 4, 30.

Lovell, B.: 1971, Quart. J. Roy. Astron. Soc. 12, 98.

Lovell, B.: 1974, Phil. Trans. Roy. Soc. London A277, 489.

Lovell, B., Maridis, L. N., and Contadakis, M. E.: 1974, Nature 250, 124.

Low, F.: 1965, IAU Circ. 1884-5.

Lutz, T. E., Furenlid, I., and Lutz, J. H.: 1973, Astrophys. J. 184, 787.

McClintock, W., Linsky, J., Gerola, H., Shine, R., Henry, R. C., and Moos, H. W.: 1974, Bull. Am. Astron. Soc. 6, 315.

McMath, R. R., Mohler, O. C., Pierce, A. K., and Goldberg, L.: 1956, Astrophys. J. 124, 1.

Martins, D. H.: 1975, Publ. Astron. Soc. Pacific 87, 163.

Matsushima, S.: 1974, Sci. Rep. Tôhoku Univ. First Ser., 57, 23.

Moffett, T. J.: 1974, Sky Telesc. 48, 94.

Moffett, T. J.: 1975a, Comm. 27 IAU, Inf. Bull. Var. Stars 995

Moffett, T. J.: 1975b, Private communication.

Moos, H. W. and Rottman, G. J.: 1972, Astrophys. J. 174, L73.

Moos, H. W., Linsky, J. L., Henry, R. C., and McClintock, W.: 1974, Astrophys. J. 188, L93.

Morton, D. C.: 1967, Astrophys. J. 150, 535.

Mullan, D. J.: 1974, Astrophys. J. 192, 149.

Mullen, D. I.: 1975, Astrophys. J. 198, 563.

Nariai, K.: 1969, Astrophys. Space Sci. 3, 150.

Oganesyan, D. B.: 1974, Soviet Astron. 17, 617.

Oster, L.: 1971, Astrophys. J. 169, 57.

Oster, L.: 1975, in B. Baschek, H. W. Kegel, and G. Traving (eds.), Problems in Stellar Atmospheres and Envelopes, Springer-Verlag, Berlin, p. 301.

Pikelner, S. B. and Khokhlova, V. L.: 1971, Comments Astrophys. Space Phys. 3, 190.

Pikeluer, S. B. and Khokhlova, V. L.: 1972, Soviet Phys. Uspekhi 15, 395.

Piotrowski, S. L., Rucinski, S. M., and Semeniuk, I.: 1974, Acta Astron. 24, 389.

Popper, D. M.: 1953, Publ. Astron. Soc. Pacific 65, 278.

Preston, G. W. and Wolff, S. C.: 1970, Astrophys. J. 160, 1071.

Preston, G. W.: 1971, Publ. Astron. Soc. Pacific 83, 571.

Reimers, D.: 1973, Astron. Astrophys. 24, 79.

Robinson, E. L. and Kraft, R. P.: 1974, Astron. J. 79, 698.

Rodonò, M.: 1974, Astron. Astrophys. 32, 337.

Rodonò, M.: 1975a, Private communication.

Rodonò, M.: 1975b, Preprint.

Rodonò, M.: 1976, This volume, p. 475.

Rosendhal, J. D.: 1973, Astrophys. J. 182, 523.

Rottman, G. J., Moos, H. W., Barry, J. R., and Henry, R. C.: 1971, Astrophys. J. 165, 661.

Schatzman, E.: 1959, IAU Symp. 9, 552.

Seaquist, E. R.: 1967, Astrophys. J. 148, L23.

Serkowski, K.: 1961, Lowell Obs. Bull. 5, 157.

Severny, A.: 1969, Nature 224, 53.

Severny, A.: 1970, Astrophys. J. 159, L73.

Sheeley, N. R. Jr.: 1967, Astrophys. J. 147, 1106.

Sitterly, B. W.: 1930, Princeton Univ. Obs. Contr. III, No. 11, p. 21.

Skumanich, A.: 1972, Astrophys. J. 171, 565.

Smith, H. J. and Smith, E. V. P.: 1963, in Solar Flares, The McMillan Company, New York, p. 270.

Struve, O.: 1952, Publ. Astron. Soc. Pacific 64, 20.

Svestka, Z.: 1970, Solar Phys. 13, 471.

Torres, C. A. O., Ferraz Mello, S., and Quast, G. R.: 1972, Astrophys. Letters 11, 13.

Torres, C. A. O. and Ferraz Mello, S.: 1973, Astron. Astrophys. 27, 231.

Tsap, T. T. and Laba, I. S.: 1973, Izv. Krynsk. Astrofiz. Obs. 48, 73.

Tuominen, J.: 1970, IAU Symp. 43, 754.

Unsöld, A.: 1955, in Physik der Sternatmosphären, 2nd edition, Springer-Verlag, Berlin, p. 606.

Unsöld, A.: 1964, Observatory 84, 152. 
Vardya, M. S.: 1970, Observatory 90, 155.

Vaughan, A. H. and Zirin, H.: 1968, Astrophys. J. 152, 123.

Vogt, S. S.: 1973, Bull. Am. Astron. Soc. 5, 399.

Vogt, S. S.: 1975, Astrophys. J. 199, 418.

Wade, C. M. and Hjellming, R. M.: 1971, Astrophys. J. 163, L105.

Walter, K.: 1971, IAU Colloq. 15, 497.

Warner, B.: 1969, Monthly Notices Roy. Astron. Soc. 144, 333.

Weiler, E. J.: 1975a, IAU Comm. 27, Inf. Bull. Var. Stars 1014.

Weiler, E. J.: 1975b, Bull. Am. Astron. Soc. 7, 267.

Weymann, R. and Chapman, G.: 1965, Astrophys. J. 142, 1268.

Wilson, O. C.: 1954, Proc. N.S.F. Conference on Stellar Atmospheres, Indiana Univ. Press, Bloomington.

Wilson, O. C. and Bappu, M. K. V.: 1957, Astrophys. J. 125, 661.

Wilson, O. C.: 1959, Astrophys. J. 130, 499.

Wilson, O. C.: 1961, Publ. Astron. Soc. Pacific 73, 15.

Wilson, O. C.: 1963, Astrophys. J. 138, 832.

Wilson, O. C. and Skumanich, A.: 1964, Astrophys. J. 140, 1401.

Wilson, O. C.: 1967, Publ. Astron. Soc. Pacific 79, 46.

Wilson, O. C.: 1968, Astrophys. J. 153, 221.

Wilson, O. C.: 1969, in S. Kumar (ed.), Low Luminosity Stars, Gordon and Breach, London, p. 103.

Wilson, O. C.: 1970, Publ. Astron. Soc. Pacific 82, 865.

Wilson, O. C. and Woolley, R.: 1970, Monthly Notices Roy. Astron. Soc. 148, 463.

Wilson, O. C.: 1976, this volume, p. 447.

Wordern, S. P.: 1974, Publ. Astron. Soc. Pacific 86, 595.

Wright, K. O.: 1973, IAU Symp. 51, 117.

\section{DISCUSSION}

Mestel: Did I understand you to say that one needs photospheric activity in an Ap star to understand the cross-over effect? Isn't this effect simply explained in the oblique rotator model as a geometrical consequence of the rotation of contiguous regions of opposite polarity?

Godoli: Yes, in order to describe the cross-over effect, we require magnetic regions of opposite polarity and the magnetic activity is the main manifestation of active regions.

Mestel: The difficulty seems to be largely semantic: you mean by photospheric activity in an Ap star just the existence of observable emerging field-lines.

Ruźdjak: Which is the highest Balmer line seen in emission in flare stars? Has the Balmer recombination continuum been observed.?

Godoli: Balmer series has been observed to $\mathrm{H}_{10}-\mathrm{H}_{14}$. As I have already said, strong emission in the Balmer continuum appears at the beginning of flares.

Schröter: I would like to add to your list of observable effects of stellar activity and its implication in interpretation one other aspect which concerns the problem of element abundances. The interaction of solar magnetic fields with photospheric and chromospheric plasma occurs apparently in two different modes; In one mode magnetic fields suppress radiation flux (sunspots, pores); in the other mode they lead to enhanced radiation (plages, network, faculae), etc. Let us apply this (so far not well understood) fact to stars with magnetic fields.

Imagine that in a stellar atmosphere many spots are present. Lines of elements with low ionization potential will occur much strengthened in these spots. Determining the abundance of elements like Li and ignoring the presence of such activity regions may well lead to an apparent overabundance of these elements. Let us now assume the surface of the star is covered considerably by features like network of faculae in which the magnetic fields cause locally enhanced radiation. Consider once again the problem of determination of the abundance of $\mathrm{Li}$ (or for example, $\mathrm{C}$ ). Ignoring the presence of these active regions shall this time lead to an apparent 'under abundance' of $\mathrm{Li}$ (or overabundance of $\mathrm{C}$ ). To my knowledge the effect of the presence of such structures has so far not been taken in account in determining element abundances (or in interpreting deviations observed in magnetic active stars).

Zwaan: Have attempts been made to find cyclic variations in the rate of flare occurrence in flare stars?

Godoli: Yes; variations have been found but not yet periodic variations.

Roxburgh: Could Dr Schröter quantify his argument on abundance determinations? If we looked at the Sun as a star what would be the error in the determination of the Lithium abundance?

Schröter: To answer your question, let us look at the behaviour of the resonance line of $\mathrm{Li}$ on the Sun. This line is almost absent in the photosphere $\left(W_{\lambda} \leq 1 \AA\right)$ and has an equivalent-width of $>200 \mathrm{~m} \AA$ in sunspots. How large the error in abundance may be when ignoring the contribution of spot-like areas to the observed spectrum depends strongly on the procedure of spectrum analysis. I can imagine that errors up to factor 10 may well arise. 